\title{
CONCORDANCE DES NEUDS DE DIMENSION 4
}

\author{
VINCENT BLANLCEIL AND OSAMU SAEKI
}

\begin{abstract}
We prove that for a simply connected closed 4-dimensional manifold, its embeddings into the sphere of dimension 6 are all concordant to each other.
\end{abstract}

\section{INTRODUCTION}

Dans cette note nous démontrons que tous les plongements dans $S^{6}$ d'une variété de dimension 4 compacte sans bord et simplement connexe sont concordants et donc cobordants.

Ce résultat complète l'étude de la concordance et du cobordisme des nœuds de dimension paire (i.e. les plongements de variétés compactes sans bord de dimension $2 n$ et $(n-1)$-connexes dans des sphères de dimension $2 n+2)$. En effet Kervaire [?] a tout d'abord démontré que le groupe de cobordisme des nœuds sphériques (i.e. les plongements de $2 n$-sphères dans des sphères en codimension 2) est trivial, et que le groupe de concordance correspondant, de dimension $2 n$, est isomorphe au groupe des classes de $h$-cobordisme des sphères d'homotopie de dimension $2 n+1$ quotienté par le sous-groupe formé des classes représentées par des sphères d'homotopie qui bordent une variété parallélisable. Ensuite dans [?, ?] on trouve une généralisation de ce résultat au monoïde commutatif des classes de cobordisme des plongements

1991 Mathematics Subject Classification. Primary 57Q45; Secondary 57Q60, 57R40, 57R65, $57 \mathrm{~N} 13$.

Key words and phrases. Concordance, cobordisme, nœud de dimension 4, chirurgie plongée. 
de variétés de dimension $2 n$ dans $S^{2 n+2}$ avec $n \geq 3$, qui sont compactes sans bord et $(n-1)$-connexes. Enfin dans [?] nous avons démontré que les classes de concordance des plongements dans $S^{4}$ d'une surface compacte sans bord et connexe, sont caractérisées par leur structure Pin ${ }^{-}$et leur nombre d'Euler du fibré normal. En particulier, deux telles surfaces plongées dans $S^{4}$ sont cobordantes si et seulement si elles sont homéomorphes abstraitement et les nombres d'Euler de leur fibré normal coïncident.

Nous nous plaçons dans la catégorie des variétés différentiables $C^{\infty}$, rappelons

Définition 1.1. Soient $M_{i}, i=0,1$, deux variétés compactes sans bord de dimension $n$ et soient $f_{i}: M_{i} \rightarrow S^{n+2}$ deux plongements.

(1) On dit que $f_{0}$ et $f_{1}$ sont concordants si $M_{0}=M_{1}=M$ et il existe un plongement propre $\Phi: M \times[0,1] \rightarrow S^{n+2} \times[0,1]$ tel que $\left.\Phi\right|_{M \times\{i\}}=f_{i}:$ $M \times\{i\} \rightarrow S^{n+2} \times\{i\}, i=0,1$.

(2) On dit que $f_{0}$ et $f_{1}$ sont cobordants (orientés) s'il existe un difféomorphisme $h: M_{1} \rightarrow M_{0}$ (qui préserve l'orientation si $M_{i}$ sont orientées) tel que $f_{0} \circ h$ et $f_{1}$ sont concordants.

\section{2. ÉNONCÉS ET DÉMONSTRATIONS}

Nous allons démontrer

Théorème 2.1. Soit $M$ une variété compacte sans bord de dimension 4 simplement connexe et orientée. Alors tous les plongements de $M$ dans $S^{6}$ sont concordants, et donc cobordants orientés. 
Démonstration. Soient $f_{i}: M \rightarrow S^{6}=S^{6} \times\{i\}, i=0,1$, deux plongements. Notons $K_{i}=f\left(M_{i}\right)$ pour $i=0,1$. On oriente les sous-variétés $K_{i}$ de $S^{6} \times\{i\}$ de telle sorte que les $f_{i}$ préservent les orientations pour $i=0,1$.

Soient $V_{i}$ des sous-variétés compactes, connexes et orientées de dimension 5 de $S^{6} \times\{i\}$ telles que $\partial V_{i}=K_{i}$ pour $i=0,1$ (c.f. [?]). Après une isotopie ambiante de $S^{6} \times\{0\}$, on peut supposer que pour une boule $\Delta \subset S^{6}$ de dimension 6 suffisamment petite, si $\Delta_{i}=\Delta \times\{i\}, i=0,1$, on a

(1) $\Delta_{0} \cap K_{0}=\Delta_{1} \cap K_{1}=\varnothing, \Delta_{0} \cap V_{0}$ coïncide avec $\Delta_{1} \cap V_{1}$ sous l'identification naturelle entre $S^{6} \times\{0\}$ et $S^{6} \times\{1\}$,

(2) $\Delta_{i} \cap V_{i}$ est difféomorphe à la boule de dimension 5 pour $i=0,1$.

Posons $V_{i}^{\prime}=V_{i} \backslash \operatorname{Int} \Delta_{i}, i=0,1$. Soit $V$ l'union de $V_{0}^{\prime}, V_{1}^{\prime}$ et du tube $\Sigma \times[0,1]$, où $\Sigma\left(\subset S^{6}\right)$ correspond à $\partial \Delta_{i} \cap V_{i}, i=0,1$, et c'est une sphère de dimension 4. Remarquons que $V$ est difféomorphe à la somme connexe orientée $V_{0} \sharp\left(-V_{1}\right)$ en dehors du bord. En outre, $B=\left(S^{6} \times[0,1]\right) \backslash(\operatorname{Int} \Delta \times[0,1])$ est une boule de dimension 7 et la variété $V$ est plongée dans $\partial B$.

Soit $W$ la variété compacte sans bord (abstraite) de dimension 5 obtenue en recollant $V$ et $M \times[0,1]$, où $\partial V=\partial V_{0} \cup \partial V_{1}$ et $M \times\{0,1\}$ sont identifiés par $f_{i}: M \times\{i\} \rightarrow \partial V_{i}, i=0,1$. Remarquons que $W$ est une variété orientable. Les sous-variétés $V_{0}$ et $V_{1}$ de $S^{6}$ sont munies de structures spin naturelles induites par celle de $S^{6}$, de plus comme $M$ est simplement connexe, la variété orientable $W$ admet une structure spin compatible avec celles de $V_{0}$ et $V_{1}$.

Comme le groupe de cobordisme des variétés spin de dimension 5 est trivial (c.f. [?] par exemple), il existe une variété spin $X$ qui est compacte et de dimension 6 telle que $\partial X=W$ en tant que variétés spin. Du fait que $\partial(M \times[0,1])=M \times\{0,1\}$, 
nous pouvons écrire

$$
\partial X=\left((V \times\{0\}) \cup_{\partial V \times\{0\}}(\partial V \times[0,1])\right) \bigcup_{f_{0} \amalg f_{1}}((M \times[0,1]) \times\{1\}),
$$

où $f_{i}:(M \times\{i\}) \times\{1\} \rightarrow K_{i} \times\{1\} \subset \partial V \times\{1\}, i=0,1$, sont des difféomorphismes d'attachement. Soit alors $\pi: \partial X \rightarrow[0,1]$ la projection sur le deuxième facteur correspondant à cette décomposition de $\partial X$. Il existe une fonction de Morse $f$ : $X \rightarrow[0,1]$ prolongeant $\pi$ et sans point critique d'indice 0 et 6 , telle que ses valeurs critiques soient dans $(\varepsilon, 1-\varepsilon)$ pour $\varepsilon>0$ un réel suffisamment petit. À l'aide de $f$, on peut donner une décomposition de $X$ par attachements d'anses sur $V \times[0,1]$ le long de $V \times\{1\}$, ceci sans utiliser des anses d'indice 0 ni 6 .

Toute 5-anse est duale d'une 1-anse. À l'aide de la réduction de Wallace (c.f. $[?, \S 6])$ on peut remplacer les 1 -anses duales par des 4 -anses, et donc remplacer les 5-anses par des 2-anses duales des 4-anses ajoutées. Ces modifications sont faites en effectuant des chirurgies spin sur $X$ sans modifier $\partial X$, ceci étant possible puisque toutes les variétés sont orientables et $M \times[0,1]$ est connexe. Ainsi on obtient une décomposition de $X$ sans 5-anse.

Considérons maintenant une 4-anse dans cette décomposition sans 5-anse, cette 4-anse est duale d'une 2-anse. Du fait que $M \times[0,1]$ est simplement connexe, le cercle d'attachement de cette 2-anse duale est trivial. De plus comme $X$ est une variété spin, attacher une 2-anse revient à faire une somme connexe le long du bord avec un $S^{2} \times D^{4}$. En utilisant à nouveau la réduction de Wallace on peut donc remplacer cette 2-anse duale par une 3-anse duale, donc par une 3-anse. Finalement on peut supposer que la décomposition en anse de $X$ ne comporte pas d'anse d'indice 4 ni 5 . 
La variété $V \times[0,1]$ est stablement parallélisable, de plus comme $X$ est spin la variété obtenue par attachement des anses d'indice 1 et 2 à $V \times[0,1]$ est elle aussi stablement parallélisable. La nullité du groupe $\pi_{2}(S O)$ prouve alors qu'après l'attachement des 3-anses la variété $X$ est encore stablement parallélisable.

Rappelons maintenant que $V$ est plongée dans le bord de la boule $B$ de dimension 7. Donc $V \times[0,1]$ est plongé dans $B$ de telle sorte que $V \times\{0\}$ corresponde à $V$ et que le produit soit transverse à $\partial B$. Comme dans [?] en effectuant les chirurgies plongées qui correspondent aux attachements des anses d'indice 1,2 et 3 sur $V \times[0,1]$ dans $B$, nous pouvons plonger $X$ dans $B$ et donc dans $S^{6} \times[0,1]$ de telle sorte que $V \times\{0\}$ corresponde à la variété $V$ de départ. La restriction de ce plongement à $M \times[0,1]$ donne alors la concordance désirée entre $f_{0}$ et $f_{1}$.

Remarque 2.2. D'après Cappell-Shaneson [?] (voir aussi [?]) une variété $M$ compacte sans bord et orientée de dimension 4 se plonge dans $S^{6}$ si et seulement si $M$ est spin et sa signature est nulle. Si de plus $M$ est simplement connexe, alors elle se plonge dans $S^{6}$ si et seulement si elle est homéomorphe à une somme connexe de copies de $S^{2} \times S^{2}$ d'après Freedman [?].

Remarque 2.3. D'après Park [?], pour des nombres impairs $m$ suffisamment grands, il existe une infinité de variétés de classe $C^{\infty}$ qui sont toutes homéomorphes à la somme connexe de $m$ copies de $S^{2} \times S^{2}$, mais qui ne sont pas difféomorphes deux à deux. Notons $\tilde{C}_{4}$ le monoïde commutatif des classes de cobordisme (orienté) des variétés de dimension 4 simplement connexes plongées dans $S^{6}$, et $\mathbf{Z}_{\geq 0}$ le monoïde commutatif des entiers positifs. Alors l'homomorphisme $\varphi: \tilde{C}_{4} \rightarrow \mathbf{Z}_{\geq 0}$ qui à une variété plongée associe la moitié du second nombre de Betti est un épimorphisme. Le résultat de Park cité ci-dessus montre que cet homomorphisme 
est loin d'être un isomorphisme, comparer aussi avec le résultat de Vogt [?, ?] dans lequel l'homomorphisme $\tilde{C}_{2 n} \rightarrow \mathbf{Z}_{\geq 0}$ correspondant pour $n \geq 3$ est un isomorphisme, où $\tilde{C}_{2 n}$ désigne le monoïde commutatif des classes de cobordisme (orienté) des variétés compactes sans bord de dimension $2 n$ et $(n-1)$-connexes plongées dans $S^{2 n+2}$.

Remarque 2.4. Lorsque $n \neq 2$, pour un plongement quelconque d'une variété compacte sans bord et orientée de dimension $2 n$ et $(n-1)$-connexe dans $S^{2 n+2}$, le plongement avec l'orientation inversée est toujours cobordant orienté au plongement original. Pour $n=2$, il existe une variété compacte $M$ sans bord de dimension 4 homéomorphe à une somme connexe de copies de $S^{2} \times S^{2}$ telle que $M$ n'est pas difféomorphe orientée à $-M$. En effet, d'après Kotschick [?], toute surface complexe compacte de type général simplement connexe qui est spin et de signature nulle convient, or une telle surface a été construite par Moishezon et Teicher [?, ?, ?]. Donc, il existe un plongement de $M$ dans $S^{6}$ qui n'est pas cobordant orienté à lui-même avec l'orientation inversée.

Remarquons de plus que pour des plongements quelconques de deux variétés homéomorphes orientées de Park dans $S^{6}$ (voir Remarque ??), on peut néanmoins trouver un $h$-cobordisme plongé dans $S^{6} \times[0,1]$ entre leurs images. Ceci se démontre comme le Théorème ?? mais en remplaçant $M \times[0,1]$ par un $h$-cobordisme arbitraire, qui existe par un résultat classique de Wall [?].

Comme corollaire on a

Corollaire 2.5. Soit $M$ une variété de dimension 4 compacte sans bord et simplement connexe plongée dans $S^{6}$. Alors il existe une variété compacte $V$ de dimension 
5 proprement plongée dans $B^{7}$ telle que $\partial V=M \subset S^{6}=\partial B^{7}$ et telle que $V$ a le type d'homotopie d'un bouquet de sphères de dimension 2 .

Démonstration. Soit $M^{\prime}$ la somme connexe des copies de $S^{2} \times S^{2}$ qui est homéomorphe à $M$. On peut supposer que $M^{\prime}$ est plongée dans $S^{6}$ de telle sorte qu'elle borde la somme connexe (le long du bord) $V^{\prime}$ des copies de $S^{2} \times D^{3}$ plongée dans $S^{6}$. Alors l'union d'un $h$-cobordisme plongé dans $S^{6} \times[0,1]$ entre $M^{\prime}$ et $M$, et $V^{\prime}$ donne la variété $V$ désirée plongée dans $S^{6} \times[0,1] \subset B^{7}$.

Comme dans [?], notre résultat peut être appliqué à la réalisation d'une décomposition (du type de Heegaard) d'une variété de dimension 5 spin compacte sans bord et simplement connexe dans $S^{7}=B^{7} \cup B^{7}$ (c.f. [?]).

En effet, toute variété $P$ compacte sans bord de dimension 5 simplement connexe et spin se décompose comme $P=H_{1} \cup H_{2}$, où $H_{1} \cong H_{2}$ est la somme connexe le long du bord d'un certain nombre de copies de $S^{2} \times D^{3}$ (c.f. [?]). Désignons par $S$ la variété $\partial H_{1}=\partial H_{2}$ de dimension 4 où $H_{1}$ et $H_{2}$ sont recollées. Soit $S^{7}=B_{1}^{7} \cup_{S^{6}} B_{2}^{7}$ la décomposition usuelle de $S^{7}$. Alors, on a

Corollaire 2.6. Soient $P=H_{1} \cup H_{2}$ et $S$ comme ci-dessus. Alors pour un plongement $\psi: S \rightarrow S^{6}$ quelconque, il existe un plongement $\Psi: P \rightarrow S^{7}=B_{1}^{7} \cup_{S^{6}} B_{2}^{7}$ qui est transverse à l'équateur $S^{6}=B_{1}^{7} \cap B_{2}^{7}$ tel que $\left.\Psi\right|_{S}=\psi$ et $\Psi^{-1}\left(B_{i}^{7}\right)=H_{i}$, $i=1,2$.

\section{REMERCIEMENTS}

Les auteurs ont bénéficié de Grant-in-Aid for Scientific Research (No. 16340018), JSPS. 


\section{REFERENCES}

[1] D. Barden, Simply connected five-manifolds, Ann. of Math. (2) 82 (1965), 365-385.

[2] V. Blanlœil et O. Saeki, Cobordisme des surfaces plongées dans $S^{4}$, à paraître dans Osaka J. Math.

[3] S. Cappell and J. Shaneson, Embeddings and immersions of four-dimensional manifolds in $\mathbf{R}^{6}$, Geometric topology (Proc. Georgia Topology Conf., Athens, Ga., 1977), pp. 301-303, Academic Press, New York, London, 1979.

[4] D. Erle, Quadratische Formen als Invarianten von Einbettungen der Kodimension 2, Topology 8 (1969), 99-114.

[5] M. Freedman, The topology of four-dimensional manifolds, J. Diff. Geom. 17 (1982), 357-453.

[6] M. A. Kervaire, Les nœuds de dimensions supérieures, Bull. Soc. Math. France 93 (1965), $225-271$.

[7] D. Kotschick, Non-trivial harmonic spinors on certain algebraic surfaces, Einstein metrics and Yang-Mills connections (Sanda, 1990), pp. 85-88, Lect. Notes in Pure and Appl. Math., Vol. 145, Dekker, New York, 1993.

[8] D. Kotschick, Orientations and geometrisations of compact complex surfaces, Bull. London Math. Soc. 29 (1997), 145-149.

[9] J. Milnor, Spin structures on manifolds, Enseignement Math. (2) 9 (1963), 198-203.

[10] B. Moishezon and M. Teicher, Existence of simply connected algebraic surfaces of general type with positive and zero indices, Proc. Nat. Acad. Sci. USA 83 (1986), 6665-6666.

[11] B. Moishezon and M. Teicher, Simply-connected algebraic surfaces of positive index, Invent. Math. 89 (1987), 601-643.

[12] J. Park, The geography of spin symplectic 4-manifolds, Math. Z. 240 (2002), 405-421.

[13] D. Ruberman, Imbedding four-manifolds and slicing links, Math. Proc. Camb. Phil. Soc. 91 (1982), 107-110.

[14] R. Vogt, Cobordismus von Knoten, "Knot theory" (Proc. Sem., Plans-sur-Bex, 1977), pp. 218-226, Lecture Notes in Math., Vol. 685, Springer-Verlag, Berlin, 1978. 
[15] R. Vogt, Cobordismus von hochzusammenhängenden Knoten, Dissertation, Rheinische Friedrich-Wilhelms-Universität, Bonn, 1978, Bonner Mathematische Schriften, 116, Universität Bonn, Mathematisches Institut, Bonn, 1980.

[16] C. T. C. Wall, On simply-connected 4-manifolds, J. London Math. Soc. 39 (1964), 141-149.

[17] A. H. Wallace, Modifications and cobounding manifolds, Canad. J. Math. 12 (1960), 503-528.

Département de Mathématiques, Université Louis Pasteur Strasbourg I, 7, Rue René Descartes, 67084 Strasbourg Cedex, France

E-mail address: blanloeil@math.u-strasbg.fr

Faculty of Mathematics, Kyushu University, Hakozaki, Fukuoka 812-8581, Japan

E-mail address: saeki@math.kyushu-u.ac.jp 